
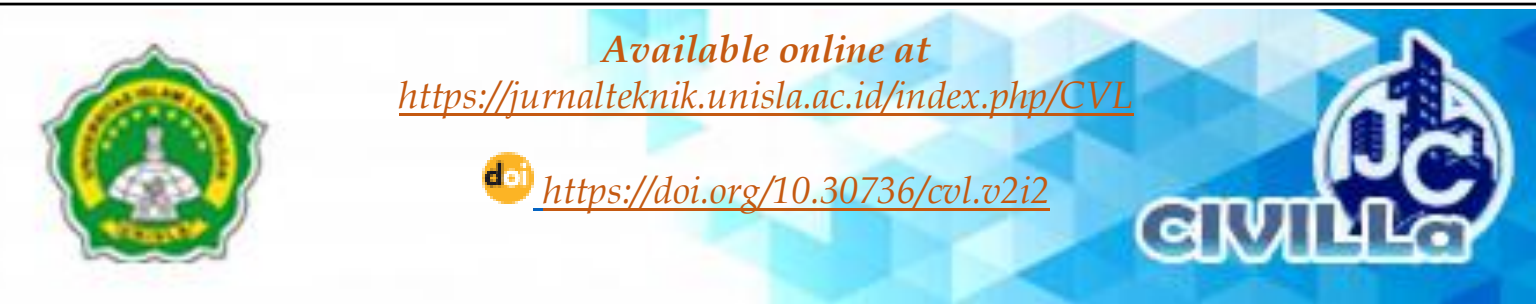

\title{
Retention Pool As Alternative For Flood Control Case Study Of Permai Satellite Housing, Sumenep Regency
}

\author{
Ach. Desmantri Rahmanto ${ }^{1}$, Faisal Rosihan ${ }^{2}$ \\ ${ }^{1,2}$, Faculty of Engineering, Universitas Wiraraja \\ Email: ${ }^{1 *}$ desmantri@wiraraja.ac.id.2 faisalrosihan5@gmail.com.
}

\section{A R T I C LE IN FO}

\section{Article History :}

Article entry : :2021-09-27

Article revised : 2021-10-16

Article received : 2021-12-09

\section{Keywords :}

Retention Pools, Flood

Mitigation, Flood

Management, Water

Infiltration.

IEEE Style in citing this article : A. D. Rahmanto and F. Rosihan, "Retention Pool As Alternative For Flood Control Case Study Of Bapertarum Housing, Sumenep Regency," CIVILA, vol. 6, no. 2, pp. 181-196, 2021.

A B S T R A C T
Problems with the environment due to development that does
not pay attention to the carrying capacity of the environment.
To overcome flooding, in this case the city of Sumenep makes
a retention pond, which is intended to reduce floods that often
occur. The capacity of the retention pond is sufficient, it can be
seen from the dimensions of the larger retention pond that the
volume of the retention pond capacity when the flood is
$6750 \mathrm{~m}^{3}>6447 \mathrm{~m}^{3}$. The retention pond is planned in the
downstream area of the residential river with a pool area of 50
x 40 meters and a depth of $4.5 \mathrm{~m}$. The bottom condition of the
pond is saturated because it is always inundated by water, so
the infiltration rate that occurs in the pond is a constant
infiltration rate $(\mathrm{fc})$. The constant infiltration for clayey soil
type is $0.5 \mathrm{~m} / \mathrm{day}$, the infiltration volume rate that occurs at the
bottom of the pond is $179 \mathrm{~m}^{3} /$ hour, the volume is below the
threshold $8283.21 \mathrm{~m}^{3}$, infiltration time is 46 hours.

Copyright (c) 2021 Ach. Desmantri Rahmanto, dkk. This work is licensed under a Creative Commons Attribution-ShareAlike 4.0 International License. Allows readers to read, download, copy, distribute, print, search, or link to the full texts of its articles and allow readers to use them for any other lawful purpose. 


\section{Introduction}

Indonesia is a water country with rivers and hundreds of deep seas. This causes Indonesia to be a country that is very prone to flooding. But basically there are several factors that cause this to happen, namely: natural events, damage to waterways and environmental degradation caused by human activities [1]. The rapid human activity in the Sumenep area has a very positive impact on economic progress. But on the other hand it can cause problems for the environment due to development that does not pay attention to the carrying capacity of the environment. To overcome flooding, in this case the City of Sumenep has created a retention pond, which is intended to reduce frequent flooding. [1] [6]. High rainfall accompanied by river conditions that experience silting due to erosion and sedimentation resulted in the river's capacity to accommodate flood discharge runoff is not optimal. This condition can cause the volume of water to overflow, resulting in a flood inundation area [6][20]. Flooding is a condition where water is not accommodated in the drain channel (times) or the flow of water is obstructed in the drain channel [17]. The hydrological cycle is a continuous process in which water moves from the earth to the atmosphere and then back to the earth again [17][18]. The length of peak flood time becomes the basis for analyzing the volume of the retention pond so that the amount of flowing retention pond discharge can be accommodated with the planned dimensions of the pond volume [1][9][11][16].

\section{Literature Review}

Flooding is a condition where water is not accommodated in the drain channel (times) or the flow of water is obstructed in the drain channel [17]. The hydrological cycle is a continuous process in which water moves from the earth to the atmosphere and then back to the earth again [18].

Retention pond is a pond that serves to temporarily hold rainwater by providing an opportunity to seep into the ground whose operations can be combined with a pump or sluice. The function of the retention pond is to replace the role of infiltration land which is used as closed land/housing/offices, so the recharge function can be replaced with a retention pond. The function of this pool is to collect direct rainwater and flow from the system to be absorbed into the ground and then can be used for water needs. [1][14].

Flood control is part of water resource management that is more specific to controlling flood discharge generally through flood control dams, or improvement of flood control carrier systems (rivers, drainage) and prevention of potentially damaging things by how to manage land use and flood areas [10]. 
Hydrological analysis, parameter testing is needed to test the suitability of the frequency distribution of the data sample to the probability distribution function which is estimated to describe or represent the frequency distribution. Parameter testing that is often used is the chi-square test and the Smirnov-Kolmogorov test [9].

To calculate the intensity of rainfall, several empirical formulas can be used such as the Talbot formula, the Sherman formula, the Ishiguro formula, and the Mononobe formula [9].

In statistics, there are several parameters related to data analysis which include the average, standard deviation, coefficient of variation and coefficient of skewness. Frequency analysis that is often used in the field of hydrology is the normal distribution, log normal distribution, Log Pearson III distribution, and Gumbel distribution [9].

The hydraulic analysis aims to determine the potential for the retention pond discharge which is calculated based on the result of the difference between the return flood discharge and the capacity discharge of each cross section of the river [3].

\section{Research Method}

\subsection{Data Collection Procedure}

The study began by analyzing the flood, namely inadequate drainage capacity. This can be done through interviews or interviews with residents.

a. Primary data

Documentation about the location to be studied, and the data from elevation measurements at the location that will be used as research samples.

b. Secondary data

Secondary data in this study is data obtained from certain parties related to the research being conducted. Certain parties, including the developer of Bapetarum Housing in the Marketing Office.

\subsection{Technical Data Analysis}

1. Planned Rain Analysis

a. Gumbel Distribution Method

$$
R_{r}=\frac{\sum R i}{n}
$$

Where :

$$
\begin{aligned}
& R_{r}=\text { Average Rain } \\
& R_{i}=\text { Rainfall } \\
& n=\text { Amount of data }
\end{aligned}
$$

b. Pearson Type III Log Method 
Calculate the logarithm of the planned rain with the selected return time, with the formula:

\section{$\log \mathrm{R} I=\log R+K \cdot S x$}

Where :

$\mathrm{R}=$ area average rain height

$\mathrm{K}=$ Pearson log frequency factor type III

Sx $=$ standard deviation

2. Rainfall Intensity

$$
\mathrm{Ii}=\frac{R_{t}}{24} \mathrm{x}\left(\frac{24}{t}\right)^{2 / 3}
$$

Where :

$$
\begin{aligned}
& \mathrm{Rt}=\text { plan rain }(\mathrm{mm}) \\
& \mathrm{t}=\text { concentration time (hour) } \\
& \text { It }=\text { Rain intensity }(\mathrm{mm})
\end{aligned}
$$

3. Flood discharge analysis

a. Rational Method

$$
\mathrm{Qt}=0,278 . \text { C.I.A }
$$

Where :

$\mathrm{Q} \mathrm{t}=$ flood discharge $\left(\mathrm{m}^{3} / \mathrm{sec}\right)$

$\mathrm{C}=$ flow coefficient

$\mathrm{I}=$ rain intensity (mm/hour)

$\mathrm{A}=$ area of flow $\left(\mathrm{km}^{2}\right)$

b. Rational modification method

$$
\mathrm{Qt}=0,278 \mathrm{C} \text {.Cs.I.A }
$$

Where :

$$
\begin{aligned}
& \mathrm{Qt}=\text { flood discharge }\left(\mathrm{m}^{3} / \mathrm{sec}\right) \\
& \mathrm{C}=\text { flow coefficient } \\
& \mathrm{Cs}=\text { deviation coefficient } \\
& \mathrm{I}=\text { rain intensity }(\mathrm{mm} / \mathrm{hour}) \\
& \mathrm{A}=\text { an area }\left(\mathrm{km}^{2}\right)
\end{aligned}
$$

4. Retention pool capacity :

$$
\mathrm{V}=\frac{1}{2} \mathrm{X}\left(\mathrm{A}_{1}+\mathrm{A}_{2}\right) \cdot \mathrm{H}
$$

Where :

$\mathrm{V}$ = storage pool volume (m3) 
$\mathrm{A} 1=$ bottom area $(\mathrm{m})$

$\mathrm{A} 2=$ top area $(\mathrm{m})$

$\mathrm{H}=$ storage pool height

\section{Results and Discussions}

\subsection{Planned Rain Analysis}

a. Gumbel Distribution

1. Ranking daily rainfall data max.

Table 1. Order CHH Max

\begin{tabular}{|c|c|}
\hline NO & CHH Max (Ri) \\
\hline 1 & 189.07 \\
\hline 2 & 177.04 \\
\hline 3 & 165.2 \\
\hline 4 & 127.70 \\
\hline 5 & 120.7 \\
\hline 6 & 103.33 \\
\hline 7 & 102.85 \\
\hline 8 & 99.71 \\
\hline 9 & 91.30 \\
\hline 10 & 66.72 \\
\hline Total & 1243.62 \\
\hline Lots of Data & 10 \\
\hline
\end{tabular}

Source:Research result (2021)

2. Determining the Average Rain Value.

$\mathrm{Rr}=\frac{\sum \mathrm{Ri}}{\mathrm{n}}=\frac{1243.64}{10}=124.40$

3. Determine the difference between the maximum rainfall and the average rain.

$(\mathrm{Ri}-\mathrm{Rr})=(189.07-124.40)=64.74$

4. Determine the difference between the maximum rainfall and the average rain. $(\mathrm{Ri}-\mathrm{Rr})^{2}=(189.07-124.40)^{2}=4182.20$

5. Determine the difference between the maximum rainfall and the average rain. $(\operatorname{Ri}-\mathrm{Rr})^{3}=(189.07-124.40)^{3}=270463.45$

6. So by following steps number 2,3 , and 4 for the following sequence, the following results are obtained. 
Table 2.Gumbel Distribution Calculation

\begin{tabular}{|c|c|c|c|c|}
\hline No & CHHMax (Ri) & $\begin{array}{c}\text { Ri- }^{-} \\
\text {Raverage }\end{array}$ & $\begin{array}{c}\left(\mathrm{Ri}^{-}\right. \\
\text {Raverage })^{2}\end{array}$ & $\begin{array}{c}(\mathrm{Ri}- \\
\text { Raverage })^{3}\end{array}$ \\
\hline 1 & 189.07 & 64.67 & 4182.20 & 270463.45 \\
\hline 2 & 177.04 & 52.64 & 2770.97 & 145863.84 \\
\hline 3 & 165.2 & 40.80 & 1664.64 & 67917.312 \\
\hline 4 & 127.70 & 3.30 & 10.89 & 35.937 \\
\hline 5 & 120.7 & -3.70 & 13.69 & -50.653 \\
\hline 6 & 103.33 & -21.07 & 443.9449 & -9353.919 \\
\hline 7 & 102.85 & -21.55 & 464.4025 & -10007.87 \\
\hline 8 & 99.71 & -24.69 & 609.5961 & -15050.93 \\
\hline 9 & 91.30 & -33.10 & 1095.61 & -36264.69 \\
\hline 10 & 66.72 & -57.68 & 3326.982 & -191900.3 \\
\hline Jumlah & 1243.62 & -0.38 & 14582.93 & 221652.13 \\
\hline
\end{tabular}

Source :Research result (2021)

7. Determining the Standard Deviation $(\mathrm{Sx})$ :

$\mathrm{Sx}=\sqrt{\frac{\sum(R i-R)^{2}}{n-1}}=\sqrt{\frac{14582.93}{9}}=40.25$

8. Calculating the Asymmetry Coefficient $\left(C_{2}\right)$

$\left(C_{2}\right)=\frac{n \cdot \sum\left(R_{i}-R_{\text {rata }}\right)^{3}}{(n-1)(n-2) s_{x}{ }^{3}}=\frac{10 \times 221652.13}{9 \times 8 \times(40.25)^{3}}=0.47$

Table 3. Gumbel Distribution Calculation

\begin{tabular}{|c|c|c|c|}
\hline Kala Ulang & Yt & Kt & Rt $(\mathbf{m m})$ \\
\hline 2 & 0.367 & -0.097 & 148 \\
\hline 5 & 1.500 & 1.096 & 222 \\
\hline 10 & 2.250 & 1.750 & 194 \\
\hline 25 & 3.199 & 2.885 & 332 \\
\hline 50 & 3.902 & 3.626 & 378 \\
\hline 100 & 4.600 & 4.361 & 423 \\
\hline
\end{tabular}

Source : Research result (2021)

9. Determine the value of $\mathrm{Yn}$ and $\mathrm{Sn}$ that depends on $\mathrm{n}$

$$
\begin{aligned}
& \mathrm{N}=10 \quad \longrightarrow \quad \mathrm{Yn}=0.5070 \\
& \mathrm{~N}=10 \quad \longrightarrow \quad \mathrm{Sn}=0.9971
\end{aligned}
$$

10. Determining the variation of the rephrase function $Y_{t}$

Function of variation for return periods 10 years $\longrightarrow Y_{t}=2.25$

11. Determine the frequency factor $(\mathrm{K})$

$$
\mathrm{K}=\frac{Y_{t}-Y_{n}}{S_{n}}=\frac{2.25-0.5070}{0.9971}=1.750
$$

12. Determine the planned rainfall with the selected return time : 


$$
\begin{aligned}
& R_{t}=R_{r}+\left(K x S_{x}\right) \\
& \mathrm{R}_{10 \text { th }}=124.40+(1.750 \times 40.25)=194.8
\end{aligned}
$$

b. Distribution of Log Person Type III

Table 4. Calculation of the Pearson Type III Log Distribution

\begin{tabular}{|c|c|c|c|c|c|}
\hline No & $\begin{array}{c}\text { CHHMax } \\
(\mathbf{R i})\end{array}$ & $\log \mathbf{R i}$ & $\log \mathbf{R i}-\log \mathbf{R}$ & $(\log \mathbf{R i}-\log \mathbf{R})$ & $(\log \mathbf{R i}-\mathbf{L o g} \mathbf{R})$ \\
\hline 1 & 189.07 & 2.277 & 0.221 & 0.253 & 0.056 \\
\hline 2 & 177.04 & 2.248 & 0.192 & 0.037 & 0.007 \\
\hline 3 & 165.2 & 2.218 & 0.162 & 0.026 & 0.004 \\
\hline 4 & 127.70 & 2.106 & 0.050 & 0.003 & 0.000 \\
\hline 5 & 120.7 & 2.082 & 0.026 & 0.001 & 0.000 \\
\hline 6 & 103.33 & 2.014 & -0.042 & 0.002 & 0.000 \\
\hline 7 & 102.85 & 2.012 & -0.044 & 0.002 & 0.000 \\
\hline 8 & 99.71 & 1.999 & -0.057 & 0.003 & 0.000 \\
\hline 9 & 91.30 & 1.960 & -0.096 & 0.009 & -0.001 \\
\hline 10 & 66.72 & 1.824 & -0.232 & 0.054 & -0.012 \\
\hline Total & 1243.62 & 20.56 & 0.18 & 0.389 & 0.054 \\
\hline
\end{tabular}

Source : Research result (2021)

1. Transform the maximum daily rainfall data to its logarithmic depth.

$\operatorname{LogRi}=\log (189.07)=2.277$

2. Calculating the middle price $(\log \mathrm{R})$

$\log \mathrm{R}=\frac{\sum \log R i}{n}=\frac{20.56}{10}=2.056$

3. $\log \mathrm{Ri}-\log \mathrm{R}=2.277-2.056=0.221$

4. $(\log R-\log R)^{2}=(0.221)^{2}=0.253$

5. $(\log \mathrm{R}-\log \mathrm{R})^{3}=(0.221)^{3}=0.056$

6. So that in a tabular manner by following steps numbers 2 to 6 for the next data, the results are as follows:

7. Calculating Standard Deviation (Sx)

$$
\mathrm{Sx}=\sqrt{\frac{\sum(\log R i-\log R i)^{2}}{n-1}}=\sqrt{\frac{0.389}{10-1}}=0.207
$$

8. Calculating the Asymmetry Coefficient $\left(\mathrm{C}_{\mathrm{s}}\right)$

$$
\mathrm{C}_{\mathrm{s}}=\frac{n \cdot \sum(\log R i-\log R)^{3}}{(n-1)(n-2) s_{x}{ }^{3}}=\frac{10 \times(0.054)}{9 \times 8 \times(0.207)^{3}}=0.919
$$

9. Determining the frequency factor $\mathrm{K}$

With data $K=0.919$ and when return periods 10 years, then return periods 10 years is $\mathrm{K}=1.339$

10. Determining the rain of the plan for the return $(\mathrm{Rt})$

$$
\log R_{1}=\log \mathrm{R}+\mathrm{K} . \mathrm{Sx}
$$




$$
\begin{aligned}
& \log R_{2}=2.056+(1.339 \times 0.207)=2.333 \\
& R_{2}=10^{2.333}=215 \mathrm{~mm}
\end{aligned}
$$

11. So that in a tabular manner by following steps numbers 3 to 8 , the results are as follows:

Table5.Planned Rainfall for Log Pearson Type III Distribution

\begin{tabular}{|c|c|c|c|c|}
\hline Kala Ulang & Log R & K & Log Rt & Rt(mm) \\
\hline 2 & 2.065 & -0.148 & 2.034 & 108 \\
\hline 5 & 2.065 & 0.769 & 2.224 & 168 \\
\hline 10 & 2.065 & 1.339 & 2.333 & 215 \\
\hline 25 & 2.065 & 2.018 & 2.483 & 304 \\
\hline 50 & 2.065 & 2.498 & 2.582 & 382 \\
\hline 100 & 2.065 & 2.957 & 2.677 & 475 \\
\hline
\end{tabular}

Source : Research result (2021)

\subsection{Rain Intensity Analysis}

Table6.Gumbel Distribution Rain Resume andLog Pearson Type III Distribution

\begin{tabular}{|c|c|c|c|c|c|c|c|}
\hline \multirow{2}{*}{$\begin{array}{c}\text { Rain } \\
\text { Station }\end{array}$} & \multirow{2}{*}{ Analysis method } & \multicolumn{5}{|c|}{ Plan Rain (mm/Day) With Return Periods } \\
\cline { 3 - 8 } & & 2 Years & 5 Years & 10 Years & 25 Years & 50 Years & 100 Years \\
\hline \multirow{2}{*}{3 Station } & Log Pearson III & 108 & 168 & 215 & 304 & 384 & 475 \\
\cline { 2 - 8 } & Gumbel & 148 & 222 & 194 & 332 & 378 & 423 \\
\hline
\end{tabular}

Source : Research result (2021)

Calculating Rain Intensity (It) Using the results of the Pearson Log method which is close to the value of $\mathrm{Cs}=0.919$, Analysis of rain intensity with various return periods.

With intervals of 2,5,10,25,50,100 years, the planned rainfall for various return periods is $108,168,215,304,384,475 \mathrm{~mm} /$ day. So for time $\mathrm{t}=10$ minutes, the rainfall intensity is:

a. Rain intensity at intervals of 25 years

$$
\begin{aligned}
\text { It } & =\frac{R_{t}}{24} \times\left(\frac{24}{t}\right)^{2 / 3} \\
\text { It } & =\frac{304}{24} \times\left(\frac{24 \times 60}{10}\right)^{2 / 3} \\
& =347 \mathrm{~mm} / \text { hour }
\end{aligned}
$$

b. Rain intensity at intervals of 50 years

$$
\begin{aligned}
\text { It } & =\frac{R_{t}}{24} \mathrm{x}\left(\frac{24}{t}\right)^{2 / 3} \\
\text { It } & =\frac{382}{24} \times\left(\frac{24 \times 60}{10}\right)^{2 / 3} \\
& =347 \mathrm{~mm} / \text { hour }
\end{aligned}
$$

c. Rain intensity at intervals of 100 years 


$$
\begin{aligned}
\text { It } & =\frac{R_{t}}{24} \mathrm{X}\left(\frac{24}{t}\right)^{2 / 3} \\
\text { It } & =\frac{475}{24} \times\left(\frac{24 \times 60}{10}\right)^{2 / 3} \\
& =543 \mathrm{~mm} / \text { hour }
\end{aligned}
$$

Table7. Rain Intensity Analysis (mm/hour)

\begin{tabular}{|c|c|c|c|c|c|c|}
\hline $\mathrm{T}$ & $\begin{array}{c}\text { lt } 2 \\
\text { Years }\end{array}$ & $\begin{array}{c}\text { lt } 5 \\
\text { Years }\end{array}$ & $\begin{array}{c}\text { lt 10 } \\
\text { Years }\end{array}$ & $\begin{array}{c}\text { lt 25 } \\
\text { Years }\end{array}$ & $\begin{array}{c}\text { lt 50 } \\
\text { Years }\end{array}$ & $\begin{array}{c}\text { It } 100 \\
\text { Years }\end{array}$ \\
\hline Minute & 108 & 168 & 215 & 304 & 382 & 475 \\
\hline 10 & 123 & 192 & 246 & 347 & 437 & 543 \\
\hline 20 & 77 & 121 & 155 & 219 & 276 & 342 \\
\hline 30 & 60 & 92 & 121 & 167 & 211 & 261 \\
\hline 40 & 50 & 76 & 99 & 138 & 174 & 215 \\
\hline 50 & 42 & 65 & 86 & 119 & 150 & 185 \\
\hline 60 & 37 & 58 & 80 & 105 & 133 & 164 \\
\hline 70 & 33 & 52 & 68 & 95 & 120 & 148 \\
\hline 80 & 30 & 48 & 62 & 86 & 109 & 135 \\
\hline 90 & 28 & 44 & 58 & 80 & 101 & 125 \\
\hline 100 & 26 & 41 & 54 & 74 & 94 & 117 \\
\hline 110 & 24 & 38 & 50 & 70 & 88 & 109 \\
\hline 120 & 23 & 36 & 48 & 66 & 83 & 109 \\
\hline 130 & 22 & 34 & 45 & 62 & 79 & 98 \\
\hline 140 & 21 & 33 & 43 & 59 & 75 & 93 \\
\hline 150 & 20 & 31 & 41 & 57 & 72 & 89 \\
\hline 160 & 19 & 30 & 39 & 54 & 62 & 85 \\
\hline 170 & 18 & 29 & 38 & 52 & 66 & 82 \\
\hline 180 & 18 & 28 & 36 & 50 & 64 & 79 \\
\hline 190 & 17 & 27 & 35 & 48 & 61 & 76 \\
\hline 200 & 16 & 26 & 34 & 47 & 59 & 73 \\
\hline 210 & 16 & 25 & 33 & 45 & 57 & 71 \\
\hline 220 & 15 & 24 & 32 & 44 & 55 & 69 \\
\hline 230 & 15 & 23 & 31 & 43 & 54 & 67 \\
\hline 240 & 14 & 23 & 30 & 41 & 52 & 65 \\
\hline S0ure & 23 & & & & \\
\hline
\end{tabular}

Source : Research result (2021)

The table above is the result of the calculation of rainfall intensity analysis with intervals of 2 years to 100 years.

\subsection{Flood Discharge Analysis}

Flood Hydrograph Rational Modification Method

A 10-year return period of 10-year return period flood discharge analysis is used with the following planning data:

1) Size of the catchment area (A) $=16,19$ ha $=0,1619 \mathrm{~km}^{2}$

2) Flow Coefficient $(\mathrm{C}) \quad=0,40$

3) Start time $\left(\mathrm{t}_{\mathrm{o}}\right) \quad=10$ minute 

4) Channel Length (L)
$=1430 \mathrm{~m}$

5) Rain plans on return periods 10 years $\left(R_{t}\right)=215 \mathrm{~mm} /$ day

6) The difference between the upstream altitude and the study location $(\Delta \mathrm{H})=0,6 \mathrm{~mm}$

Calculation:

a). Average flow rate:

$$
\mathrm{V}=72\left(\frac{\Delta \mathrm{H}}{L}\right)^{0,6}=72\left(\frac{0,6}{1.430}\right)^{0,6}=0.67 \mathrm{~m} / \mathrm{sec}
$$

b). Flow time along the river:

$$
\mathrm{t}_{\mathrm{d}}=\frac{\mathrm{L}}{60 \mathrm{~V}}=\frac{1.430}{60 \times 0,67}=35 \text { minute }
$$

c). Concentration Time

$$
\mathrm{t}_{\mathrm{c}}=\mathrm{t}_{\mathrm{o}}+t_{d}=10+35=45 \text { minute }
$$

d). Deviation coefficient

$$
\mathrm{C}_{\mathrm{S}}=\frac{2 t_{c}}{2 t_{c}+t_{d}}=\frac{2 \times 45}{(2 \times 45)+35}=0,72
$$

e). Deviation coefficient:

$$
I_{t}=\frac{R_{t}}{24} \times\left(\frac{24}{t}\right)^{2 / 3}=\frac{215}{24} \times\left(\frac{24 \times 60}{45}\right)^{2 / 3}=90 \mathrm{~mm} / \mathrm{hour}
$$

f). Incoming water discharge

$$
\begin{aligned}
Q_{\text {in }} & =0,278 \times C \times C_{s} \times I_{t} \times A \\
& =0,278 \times 0,40 \times 0,72 \times 90 \times 0,1619=1,16 \mathrm{~m} / \mathrm{sec}
\end{aligned}
$$

1. Calculation of retention pond volume and pump capacity:
a. Flow time along the river $\left(t_{d}\right)$
$=35$ minute
b. Concentration time $\left(\mathrm{t}_{\mathrm{c}}\right)$
$=45$ minute
c. Rain plan for 10 year return period $\left(\mathrm{R}_{\mathrm{t}}\right)$
$=215 \mathrm{~mm} /$ day
d. Rain intensity (I)
$=137 \mathrm{~mm} / \mathrm{hour}$
e. Incoming water discharge $\left(Q_{\text {in }}\right)$
$=1,16 \mathrm{~m}^{3} / \mathrm{sec}$

2. The cumulative inflow volume calculation from the graph above is as follows:

Table 8. Cumulative Flow $Q_{\text {in }}$ Dimensions $t_{c}$

\begin{tabular}{|c|c|c|c|c|c|}
\hline Cumulative time(minute) & $\begin{array}{c}\text { Inflow } \\
\left(\mathbf{m}^{\mathbf{3}} / \mathbf{s e c}\right)\end{array}$ & $\begin{array}{c}\text { AverageInflow } \\
\left(\mathbf{m}^{\mathbf{3}} / \mathbf{s e c}\right)\end{array}$ & $\mathbf{A t}$ & $\begin{array}{c}\text { Volume } \\
(\mathbf{m 3})\end{array}$ & $\begin{array}{c}\text { Cumulative } \\
\text { volume 1 }\end{array}$ \\
\hline 10 & 0.31 & 0.16 & 600 & 93 & 93 \\
\hline 20 & 0.55 & 0.43 & 600 & 258 & 351 \\
\hline 30 & 0.81 & 0.68 & 600 & 408 & 759 \\
\hline 40 & 1.1 & 0.96 & 600 & 573 & 1332 \\
\hline 45 & 1.23 & 1.17 & 600 & 699 & 2031 \\
\hline 50 & 1.15 & 1.19 & 600 & 714 & 2745 \\
\hline 60 & 0.99 & 1.07 & 600 & 642 & 3387 \\
\hline 70 & 0.79 & 0.89 & 600 & 534 & 3921 \\
\hline
\end{tabular}




\begin{tabular}{|c|c|c|c|c|c|}
80 & 0.71 & 0.75 & 600 & 450 & 4371 \\
\hline 90 & 0.51 & 0.61 & 600 & 366 & 4737 \\
\hline 100 & 0.29 & 0.40 & 600 & 240 & 4977 \\
\hline 110 & 0.21 & 0.25 & 600 & 150 & 5127 \\
\hline 120 & 0 & 0.11 & 600 & 63 & 5190 \\
\hline 130 & 0 & 0.00 & 600 & 0 & 5190 \\
\hline
\end{tabular}

Source : Research result (2021)

Example Calculation table above:

a. Data taken from 45 minutes

$$
\text { rInflow } \quad=\frac{\text { Inflow }+ \text { Inflow }}{2}=\frac{1.10+1.23}{2}=1.17 \mathrm{~m}^{3} / \mathrm{sec}
$$

b. $\mathrm{V}=\mathrm{r}$ inflow $\mathrm{x}$ At

$$
=1.17 \times 600=699 \mathrm{~m}^{3}
$$

c. $\mathrm{KV}=\mathrm{KV}+\mathrm{V}$

$$
=1332+699=2031 \mathrm{~m}^{3}
$$

3. Calculation of critical inflow capacity by trying (trial \& error) hydrograph model of critical retention pond conditions $t_{e}>t_{c}$

Tired : return periods 10 yearswitht $_{\mathrm{c}}=60$ minutes, $\mathrm{i}=80 \mathrm{~mm} / \mathrm{hour}$.

$$
\begin{aligned}
\mathrm{C}_{\mathrm{s}} & =\frac{2 t_{c}}{2 t_{c}+t_{d}}=\frac{2 \times 60}{(2 \times 60)+35}=0,77 \\
\text { Qin' }_{\text {in }} & =0,278 \text { C.C. } .1 . \mathrm{A} \\
& =0,278 \times 0,40 \times 0,77 \times 80 \times 0,1619 \quad=1,10 \mathrm{~m}^{3} / \mathrm{sec}
\end{aligned}
$$

The cumulative calculation of the inlet volume from the graph above is as follows :

Table 9. Cumulative Inflow Volume Q $_{\text {indimensionst }}$ Critical

\begin{tabular}{|c|c|c|c|c|c|}
\hline $\begin{array}{c}\text { Cumulative Time } \\
(\text { Minute })\end{array}$ & $\begin{array}{c}\text { Inflow } \\
(\mathbf{m 3 / S e c})\end{array}$ & $\begin{array}{c}\text { Average inflow } \\
(\mathbf{m 3 / S e c})\end{array}$ & At & $\begin{array}{c}\text { Volume } \\
(\mathbf{m 3})\end{array}$ & $\begin{array}{c}\text { Cumulative } \\
\text { volume 1 } \\
(\mathbf{m 3})\end{array}$ \\
\hline 0 & 0 & 0.15 & 600 & 87 & 87 \\
\hline 10 & 0.29 & 0.40 & 600 & 237 & 324 \\
\hline 20 & 0.5 & 0.61 & 600 & 366 & 690 \\
\hline 30 & 0.72 & 0.87 & 600 & 519 & 1209 \\
\hline 40 & 1.01 & 1.06 & 600 & 633 & 1842 \\
\hline 45 & 1.1 & 1.11 & 600 & 666 & 2508 \\
\hline 50 & 1.12 & 1.11 & 600 & 666 & 3174 \\
\hline 60 & 1.1 & 1.05 & 600 & 627 & 3801 \\
\hline 70 & 0.99 & 0.97 & 600 & 579 & 4380 \\
\hline 80 & 0.94 & 0.87 & 600 & 522 & 4902 \\
\hline 90 & 0.8 & 0.74 & 600 & 444 & 5346 \\
\hline 100 & 0.68 & 0.67 & 600 & 399 & 5745 \\
\hline 110 & 0.65 & 0.53 & 600 & 315 & 6060 \\
\hline 120 & 0.4 & 0.36 & 600 & 213 & 6273 \\
\hline 130 & 0.31 & & & \\
\hline
\end{tabular}




\begin{tabular}{|c|c|c|c|c|c|}
\hline 140 & 0.21 & 0.26 & 600 & 156 & 6429 \\
\hline 150 & 0.1 & 0.16 & 600 & 93 & 6522 \\
\hline 155 & 0 & 0.05 & 600 & 30 & 6552 \\
\hline 160 & 0 & 0.00 & 600 & 0 & 6552 \\
\hline
\end{tabular}

Source : Research result (2021)

Example Calculation table above:

d. Data taken from 45 minutes

$$
\text { rinflow }=\frac{\text { inflow }+ \text { inflow }}{2}=\frac{1.10+1.01}{2}=1.06 \mathrm{~m}^{3} / \mathrm{sec}
$$

a. $\mathrm{V}=\mathrm{r}$ inflow $\mathrm{x}$ At

$$
=1.06 \times 600=633 \mathrm{~m}^{3}
$$

b. $\mathrm{KV}=\mathrm{KV}+\mathrm{V}$

$$
=1209+633=1842 \mathrm{~m}^{3}
$$

\subsection{Pool Infiltration Capacity}

1. Retention Pool Volume

The dimensions of the retention pond are planned as follows:

$$
\begin{aligned}
& A 1=2000 \mathrm{~m} \\
& A 2=1000 \mathrm{~m} \\
& H=4,5
\end{aligned}
$$

From the data above, the dimensions of the retention pool will be calculated:

$$
\begin{aligned}
\mathrm{V} & =\frac{1}{2} \mathrm{x}\left(A_{1}+A_{2}\right) \cdot \mathrm{H} \\
\mathrm{V} & =\frac{1}{2} \mathrm{x}(2000+1000) \cdot 4,5=6750 \mathrm{~m}^{3}
\end{aligned}
$$

So the capacity of the retention pond is sufficient because the dimensions of the retention pond are larger than the volume of the retention pond capacity when the flood is $6750 \mathrm{~m}^{3}>6447 \mathrm{~m}^{3}$

2. Infiltration

The retention pond is planned in the downstream area of the residential river with a pool area of $50 \times 40$ meters and a depth of $4.5 \mathrm{~m}$. The bottom condition of the pond is saturated because it is always inundated by water, so the infiltration rate that occurs in the pond is a constant infiltration rate (fc). Infiltration constant for loamy clay is $0.5 \mathrm{~m} /$ day

$\mathrm{Fc}=0,5 \mathrm{~m} /$ day $=\frac{0,5}{24}=0,02083 \mathrm{~m} /$ hour

The rate of infiltration volume that occurs at the bottom of the pond:

$$
\begin{aligned}
\mathrm{V} & =\text { fc } \times \text { A pool } \\
\mathrm{V} & =0,02083 \times 8631,4695=179 \mathrm{~m}^{3} / \text { hour }
\end{aligned}
$$


Volume below threshold:

$$
\begin{aligned}
& \mathrm{V}=\frac{1}{2} \times\left(A_{1}+A_{2}\right) . \mathrm{H} \text {. pool length } \\
& \mathrm{V}=\frac{1}{2} \times(39,1128+34,5158) \times 4,5 \times 50=8283,21 \mathrm{~m}^{3} \\
& \text { Infiltration Time: } \\
& \mathrm{V}=\mathrm{v} \times \mathrm{t} \\
& \mathrm{T}=\mathrm{v} / \mathrm{v} \\
& \quad=8283,21 / 179 \quad=46 \text { hour }
\end{aligned}
$$

Within 46 hours the water from the retention pond has seeped into the ground.

\section{Conclusion and Suggestion}

\subsection{Conclusion}

The capacity of the retention pond is sufficient, it can be seen from the dimensions of the larger retention pond that the volume of the retention pond capacity when the flood is $6750 \mathrm{~m}^{3}>6447 \mathrm{~m}^{3}$. The retention pond is planned in the downstream area of the residential river with a pool area of $50 \times 40$ meters and a depth of $4.5 \mathrm{~m}$. The bottom condition of the pond is saturated because it is always inundated by water, so the infiltration rate that occurs in the pond is a constant infiltration rate (fc). The constant infiltration for clayey soil type is $0.5 \mathrm{~m} / \mathrm{day}$, the infiltration volume rate that occurs at the bottom of the pond is $179 \mathrm{~m}^{3} /$ hour, the volume is below the threshold $8283.21 \mathrm{~m}^{3}$, infiltration time is 46 hours.

\subsection{Suggestion}

Some suggestions that can be submitted after this research is carried out are:

1. There needs to be serious handling of conditions where the main channel at the location is smaller than the discharge that must be accepted.

2. It takes awareness to protect the environment from local residents. One of them is not to throw garbage in channels that should be crossed by water.

\section{References}

[1] Amin, M. (2016). Analisis Genangan Banjir di Kawasan Sekitar Kolam Retensi dan Rencana Pengendaliannya, Studi Kasus: Kolam Retensi Siti Khadijah Palembang. Jurnal Perencanaan Wilayah Dan Kota, Volume 2, No 2.

[2] Anang Bagus Prakoso. 2017. Perencanaan Kolam Retensi Untuk penanggulangan Banjir Kecamatan Tembalang.

[3] Astuti, D. dkk. (2015). Analisis kolam retensi sebagai pengendalian banjir Genangan di kecamatan payung sekaki. JOMFTEKNIK, Volume 3, No 1. 
[4] Direktorat Jendral Cipta Karya. (2010). Tata Cara Pembuatan Kolam Retensi dan Polder. Jakarta: Departemen Pekerjaan Umum.

[5] Departemen Pemukiman dan Prasarana Wilayah (Direktorat Jenderal Tata Perkotaan dan Perdesaan). 2003. Panduan dan Petunjuk Praktis Pengelolaan Drainase Perkotaan. Jakarta.

[6] Ervina Wulandari abdillah. 2014 Perencanaan Sistem Drainase Melalui Metode Sumurb resapan komunal pada Perumahan Bumi Sumekar Asri Kabupaten Sumenep

[7] Febrinasti alia, Rhaptyalyani, dan ReiniSilvi Ilmiaty. April 2018. Perencanaan Kolam Retensi Pengendalian Banjir Di RSMH Kota Palembang.

[8] Kamiana I. Made, 2011. Teknik Perhitungan Debit Rencana Bangunan Air, Graha Ilmu, Yogyakarta.

[9] Florince, F. dkk. (2015). Studi Kolam Retensi sebagai Upaya Pengendalian Banjir Sungai Way Simpur Kelurahan Palapa Kecamatan Tanjung Karang Pusat. JRSDD, Volume 3, Hal: 507-520.

[10] Kodoatie, Robert J., dan Sugiyanto. 2002. Banjir - Beberapa Penyebab dan Metode Pengendaliannya dalam Perspektif Lingkungan. Cetakan 1, Pustaka Pelajar:Yogyakarta.

[11] Limantara Lily Montarcih, 2010. Hidrologi Praktis, Lubuk Agung, Bandung. Suryadinata G, 2013, Evaluasi Rencana Kinerja Kolam Retensi (Retarding Basin) Dalam Upaya Pengendalian Banjir Tukad Matid di Kota Denpasar, (Jurnal), Departemen Teknik Sipil, Universitas Udayana, Denpasar.

[12] L.Samang, 2014. Typikal Drainase Retensi Pengendali Banjir Zona Jl.Sulawesi dan Sekitarnya Kota Makassar, (Jurnal), Jurusan Sipil Fakultas Teknik Universitas Hasanuddin, Makassar.

[13] Mohamad Lutfi, Fadhila Muhammad Libasut Taqwa. 2014. Indentifikasi Lahan Resapan Dalam Penanganan Banjir Di Kawasan Simpang Johor-Yasmin

[14] Peraturan Menteri Pekerjaan Umum Nomor 12/Prt/M/2014 Tentang Penyelenggaraan Sistem Drainase Perkotaan. 2014. Tata Cara Perencanaan Sistem Drainase Perkotaan.

[15] Soewarno, 1997. Hidrologi Pengukuran dan Pengelolaan Data Aliran Sungai (Hidrometer), NOVA, Bandung.

[16] Sumanto, (2018). Penerapan Sistem Kolam Retensi (Retanding Basin) Pada Daerah Aliran Sungai Deli Untuk Pengendalian Banjir Kota Medan.

[17] Suripin, 2004. Sistem Drainase Perkotaan yang Berkelanjutan, Andi Offset, Yogyakarta

[18] Triatmodjo, Bambang. 2008. Hidrologi Terapan. Beta Offset, Yogyakarta.

[19] Wesli. 2008. Drainase Perkotaan. Graha Ilmu. Yogyakarta. 
Civilla : Jurnal Teknik Sipil Universitas Islam Lamongan

ISSN (Online) 2620-7222

Volume 06 Number 2 Year 2021

ISSN (Print) 2503-2399

[20] Zevri Azril, (2017). Analisis Volume Tampungan Kolam Retensi Das Deli Sebagai Salah

Satu Upaya Pengendalian Banjir Kota Meda. Jurnal Rekayasa Sipil, Volume 13 No. 2 